\title{
Prevalence and Clinical Profile of EGFR Mutation In Non- Small-Cell Lung Carcinoma Patients in Southwest China
}

\author{
Juan Zhou, Xing-Bo Song, He He, Yi Zhou, Xiao-Jun Lu*, Bin-Wu Ying*
}

\begin{abstract}
Aims: To investigate the distribution of epidermal growth factor receptor (EGFR) mutations, and explore any relationships with clinical characteristics in non-small-cell lung carcinoma (NSCLC) patients. Materials and Methods: EGFR mutations were assessed by ADx-ARMS in 261 NSCLC patients from West China Hospital of Sichuan University. Relationships between EGFR mutation and clinical characteristics were analyzed by SPSS. Results: The EGFR mutation rate was 48.7\% (127/261), 19-del and L858R mutations occurred predominantly, accounting for $33.1 \%$ and $40.9 \%$, respectively, in mutated cases. Moreover, $10.2 \%$ patients were found to carry double mutations. EGFR mutations occurred more frequently in women $(57.5 \%)$ than in men $(41.8 \%)(P=0.01)$, and were more frequent in non-smokers $(61.2 \%)$ than in former or current smokers $(31.2 \%)(P<0.00)$. In addition, they were more common in adenocarcinomas $(52.8 \%)$ and adenosquamous carcinomas $(\mathbf{4 2 . 8 \%})$ than in squamous cell carcinomas $(\mathbf{1 4 . 8 \%})(p<0.00)$. However, only smoking history and pathological types, rather than gender, proved to be associated with EGFR mutations on multivariate logistic regression analysis. No significant differences in pathological stage and metastasis status were found between EGFR wild-type and mutated cases, although EGFR mutation type was related to pathological type $(p=0.00)$ - 19-del, L858R and other mutation types respectively occurred in $34.2 \%, 42.5 \%$ and $23.3 \%$ of adenocarcinomas, but in $14.3 \%, 0 \%$ and $85.7 \%$ of non-adenocarcinomas. Conclusions: The EGFR mutation rate was $48.7 \%$ in NSCLCs in Southwest China, so that nearly $40 \%$ patients might benefit from targeted therapies. Smoking status and pathological types were independent predictors of EGFR mutation, while EGFR mutation type was related to only pathological type, rather than smoking status.
\end{abstract}

Keywords: Non-small-cell lung carcinoma - mutation - epidermal growth factor receptor - Southwest China

Asian Pac J Cancer Prev, 17 (3), 965-971

\section{Introduction}

Lung cancer is one of the most common cancers in the world, with sharply increasing morbidity and mortality in the last decades. It was estimated that about 1.8 million new lung cancer cases occurred in 2012 worldwide by GLOBOCAN, occupied $13 \%$ of all cancers, with only $15 \%$ five-year survival rate. It has become the leading cause of cancer death among males in the whole world, and has surpassed breast cancer as the leading cause of cancer death among females in more developed countries (Torre et al., 2015). Even worse, the morbidity and mortality were both higher in China (National Office for Cancer Prevention and Control, 2010; She et al., 2013). According to WHO classification of lung cancer, nonsmall-cell lung cancer (NSCLC) is the main type of lung cancer, occupied 80-85\%, including Adenocarcinoma, squamous carcinoma and adenosquamous carcinoma, et al. NSCLC is a cancer with high malignancy, 30-40\% cases are diagnosed at the advanced stage, without the opportunity of operation, and the effect of chemotherapy is limited. With the development of molecular mechanism research in lung cancer, some gene mutations and genetic recombinations were found to be involved in NSCLC pathogenesis, including KRAS, EGFR, ALK, ROS 1, et al, which provided new treatment protocols, pushing NSCLC into a new era of targeted therapy (Pao and Hutchinson, 2012).

EGFR has become a hotspot target in NSCLC therapy development in recent years. EGFR gene locates in the 7p12-14 region, encoding a member of the receptor tyrosine kinase (RTK) family, which forms receptor heterologous or homologous dimers on cell surface after combination with corresponding ligands, leading to specific tyrosine residues phosphorylation, to regulate PI3K/AKT, ERK/MAPK and STAT pathways, and finally participate in cell proliferation, apoptosis and angiogenesis (Eck and Yun, 2010; Yasuda et al., 2012). Over expression of EGFR or EGFR ligand, activation by EGFR mutation were reported to induce carcinogenesis, and the latter is the major cause of abnormal biological behavior of tumor cells, which could enhance the effect of receptor and 
prolong the function duration (Lynch et al., 2004). EGFR tyrosine kinase inhibitors (TKIs) such as Gefitinib and Erlotinib, could competitive inhibit combination of ATP with kinase catalytic site in EGFR intracellular region, thus inhibit EGFR tyrosine kinase activity, blocking EGFR signal pathway, and finally target cancer cells. Previous researches indicated that EGFR-TKIs could significantly prolong PFS and OS in patients with EGFR mutation (Shepherd et al., 2005; Mok et al., 2009). IPASS (Iressa Pan-Asian Study) reported that the response rate in the patients with gefitinib was much higher than those with carboplatin-paclitaxel $(71.2 \%$ versus $47.3 \%)$ in the mutation-positive subgroup, while much lower $(1.1 \%$ versus $23.5 \%$ ) in the mutation-negative subgroup (Mok et al., 2009). In summary, it was commonly recognized that only patients with EGFR mutation would benefit from EGFR-TKIs.

EGFR gene contains 28 exons, mutations often occurre in the EGFR tyrosine kinase enconding region, focused on exons $18-21$, account for more than $90 \%$ of all mutation types, and deletions in exon 19 and L858R in exon 21 are most common, which are considered as sensitive mutations. However, patients with different mutation type obtained different efficacy, for example, patients with deletions in exon 19 achieved higher effective rate and longer survival time than patients with L858R mutation after erlotinib or gefitinib therapy (Mitsudomi et al., 2005); D770-N771 mutation in exon 20 was reported to be less sensitive to EGFR-TKIs (Kobayashi et al., 2005); T790M mutation in exon 20 could change the structure of ATP-binding pocket, generating steric effect when TKI bind with kinase region, thus leads to TKI resistance (Doss et al., 2014). So, knowledge of EGFR mutation type is as essential as EGFR mutation status in the NSCLC individualized treatment.

EGFR mutation rate varies in different districts worldwide, from $10 \%$ to $20 \%$ in west world, while from $30 \%$ to $50 \%$ in Asia. However, there were few reports about EGFR mutation profile of NSCLC patients in Southwest China. Besides, relationship between EGFR mutation and clinical characteristics of NSCLC remains controversial (Gu et al., 2007; Wu et al., 2007; Liam et al., 2014; Shi et al., 2014; Tian et al., 2014; Zhao et al., 2014; Zheng et al., 2014). So, this study was conducted to reveal the EGFR mutation profile of NSCLC patients in Southwest China, and explore the relationship between EGFR mutation and clinical characteristics, including age, gender, smoking history, pathological types, and pathological stage.

\section{Materials and Methods}

\section{Subjects and samples}

261 cases with pathologically confirmed NSCLC patients were enrolled from West China Hospital, including 148 males and 113 females, the average age was $58.3 \pm 11.5$ years. Pathological types and pathological stage (pTNM) were determined according to WHO tumor classification and diagnostic criteria. Biopsy or pleural fluid samples were collected, and detected by a pathologist to have enough cancer cells. Written informed consents were obtained from all included individuals and approval for this study was obtained from the ethical committee of West China Hospital, Sichuan University.

\section{EGFR mutation detection}

DNA was extracted by QIAamp DNA FFPE Tissue kit (QIAGEN) according to the instructions, and then the DNA purity and concentration were detected by NanoDrop 2000 spectrophotometer (Thermo Fisher), with A260/A280 limited to 1.8-2.0, and then adjusted the concentration to $1.5-3 \mathrm{ng} / \mu \mathrm{l}$.

Amplification refractory mutation system (ARMS) was used to detect EGFR mutation, with ADx-ARMS ${ }^{\circledR}$ EGFR mutation detection kit (AmoyDx) following instructions, simultaneously detecting 29 mutations of EGFR, including 19-del (19 deletions in exon 19), 20ins (3 insertions in exon 20), G719X (including G719S, G719A, G719C), L858R, L861Q, S7681, and T790M. External control in every sample and internal control in every tube were used to avoid effect of DNA insufficiency or PCR inhibitors.

\section{Statistics}

Means Testing was used to compare age of EGFR mutation group with wild-type group. Pearson Chi-square or Fisher exact test were used to analyze the relationship between EGFR mutation and characteristics of NSCLC, including gender, smoking history, pathological types, and pathological stage. Furthermore, logistic stepwise regression was used to rule out the impact of confounding factors. All the statistics were performed by SPSS 17.0, $P<0.05$ was defined as significant with two-sided test.

\section{Results}

EGFR mutation distribution of NSCLC patients in Southwest China

EGFR mutations were found in 127 cases out of 261 NSCLC patients, accounted for $48.7 \%$ mutation rate. L858R and 19-del were the major types, accounted for $40.9 \%$ (52 cases) and $33.1 \%$ (42 cases) of all the mutated cases respectively; T790M mutation took the third place, accounted for $6.3 \%$ ( 8 cases); The rest mutation types were rare, accounted for 9.4\% (12 cases), including 20-INS, G719C, G719S, L861Q and S768I, as shown in Table 1. Moreover, sensitive mutations accounted for

Table 1. EGFR Mutation Distribution of NSCLC Patients in Southwest China

\begin{tabular}{lrr}
\hline Mutation type & Cases & \multicolumn{1}{c}{ Mutation rate } \\
\hline Sensitive mutations & 102 & $39.1 \%(102 / 261)$ \\
19-del & 42 & $33.1 \%(42 / 127)$ \\
L858R & 52 & $40.9 \%(52 / 127)$ \\
G719C or G719S & 2 & $0.9 \%(2 / 127)$ \\
L861Q or S768I & 6 & $4.7 \%(6 / 127)$ \\
Resistant mutations & 12 & $4.6 \%(12 / 261)$ \\
T790M & 8 & $6.3 \%(8 / 127)$ \\
20-INS & 4 & $3.1 \%(4 / 127)$ \\
Double mutations & 13 & $10.2 \%(13 / 127)$ \\
\hline Total & 127 & $48.7 \%(127 / 261)$ \\
\hline
\end{tabular}


Table 2. Mutation Patterns of 13 Cases with Double Mutations

\begin{tabular}{|c|c|c|c|c|c|c|c|c|c|c|c|}
\hline Patient & $\begin{array}{l}19- \\
\text { del }\end{array}$ & L858R & T790M & $\begin{array}{l}20- \\
\text { INS }\end{array}$ & $\begin{array}{c}\text { G719C } \\
\text { or } \\
\text { G719S }\end{array}$ & $\begin{array}{c}\text { L861Q } \\
\text { or } \\
\text { S768I }\end{array}$ & Gender* & $\begin{array}{l}\text { Smoking } \\
\text { history** }\end{array}$ & $\begin{array}{l}\text { Pathological } \\
\text { types*** }\end{array}$ & $\begin{array}{l}\text { TNM } \\
\text { stage }\end{array}$ & $\begin{array}{l}\text { Pathological } \\
\text { stage }\end{array}$ \\
\hline 1 & + & - & + & - & - & - & $\mathrm{F}$ & $\mathrm{N}$ & A & T2N2M1 & IV \\
\hline 2 & + & - & - & - & + & - & F & $\mathrm{N}$ & A & T3NIM1 & IV \\
\hline 3 & + & - & - & - & - & + & F & $\mathrm{N}$ & A & $\mathrm{T} 2 \mathrm{~N} 2 \mathrm{M} 0$ & I \\
\hline 4 & - & + & + & - & - & - & F & $\mathrm{N}$ & S & T3N2M0 & III \\
\hline 5 & - & + & + & - & - & - & F & $\mathrm{N}$ & A & $\mathrm{T} 2 \mathrm{~N} 2 \mathrm{M} 0$ & III \\
\hline 6 & - & + & + & - & - & - & M & $\mathrm{N}$ & A & $\mathrm{T} 2 \mathrm{~N} 3 \mathrm{MX}$ & III \\
\hline 7 & - & + & + & - & - & - & M & $\mathrm{Y}$ & A & T4N2M0 & III \\
\hline 8 & - & + & + & - & - & - & M & $\mathrm{N}$ & S & T4N2M1 & IV \\
\hline 9 & - & + & + & - & - & - & M & $\mathrm{Y}$ & A & T4N3M1 & IV \\
\hline 10 & - & + & - & - & - & + & F & $\mathrm{N}$ & A & T3N1M0 & III \\
\hline 11 & - & - & + & + & - & - & M & $\mathrm{Y}$ & A & T4N3M1 & IV \\
\hline 12 & - & - & + & - & - & + & M & Y & A & T2N0M0 & I \\
\hline 13 & - & - & + & - & - & + & M & $\mathrm{N}$ & A & T1N1M1 & IV \\
\hline
\end{tabular}

PS:* F: female, M: male; ** N: no, no smoker; Y: yes, former of current smoker; *** A: adenocarcinoma; S: squamous carcinoma

$39.1 \%(102 / 261)$ of all the patients, including 19-del, L858R, G719C, G719S, L861Q and S768I, while resistant mutations accounted for $4.6 \%$ (12/261), including T790M and $20-\mathrm{INS}$.

Mutation patterns and clinical characteristics of 13 cases with double mutations

13 cases $(10.2 \%)$ were found to contain double mutations, as shown in table $2, \mathrm{~F} / \mathrm{M}$ was $6 / 7$, smoking rate was $30.8 \%$ (4/13), adenocarcinoma patients accounted for $84.6 \%(11 / 13)$, pathological stageI, III, IV accounted for $15.4 \%, 38.5 \%, 46.2 \%$ respectively, similar to the whole group (as shown in Table 3. Line "Total"). 7 mutation patterns were found, of which L858R combined with T790M occurred most common, accounted for $46.2 \%$ (6 cases). Interestingly, mutation pattern carrying T790M accounted for $76.9 \%$ (10/13), which was recognized as a common secondary drug-resistant mutation.

Correlation analysis of EGFR mutation status and characteristics of NSCLC patients

We compared age, gender, smoking history, pathological types, and pathological stage between EGFR mutated and wild-type patients, EGFR mutation rate was found to be related with gender, smoking history, and pathological types, as shown in Table 3.

No significant difference of average age was found between patients with EGFR mutation $(58.8 \pm 10.6$ years $)$ and those without EGFR mutation $(57.8 \pm 12.2$ years) $(p=0.49)$; EGFR mutation rate was higher in females $(57.5 \%, 65 / 113)$ than in males $(41.9 \%, 62 / 148)(p=0.01)$; It was also higher in patients without smoking history $(61.2 \%, 93 / 152)$ than patients with smoking history $(31.2 \%, 34 / 109)(p<0.00)$; Fisher exact testing revealed significant difference of EGFR mutation rate among subgroups classified by pathological types $(p<0.00)$, EGFR mutation rate of adenocarcinoma patients was highest, accounted for $52.8 \%$ (120/227), mutation rate of adenosquamous carcinoma patients took the second place $(42.8 \%, 3 / 7)$, and mutation rate of squamous carcinoma patients was lowest $(14.8 \%, 4 / 27)$.

Correlation analysis of EGFR mutation status with pathological stage showed no significant difference of EGFR mutation rate between subgroups classified by either $P$ stage or TNM stage $(p>0.05)$. Meanwhile, we could find that EGFR mutation rates were similar in patients with or without lymphatic metastasis (N0 vs $\mathrm{N} 1+\mathrm{N} 2+\mathrm{N} 3)\left(\mathrm{x}^{2}=0.15, P=0.69\right)$, which accounted for $48.0 \%$ (94/196) and 50.8\% (33/65) respectively; Distant metastasis was uncorrelated with EGFR mutation status either (M0 vs M1) $\left(\mathrm{x}^{2}=0.01, P=0.94\right)$.

However, we found that smoking history was closely related with gender $(P<0.00)$, smoking history occurred more often in males than in females $(68.2 \%$ vs $7.1 \%)$. To rule out the impact of confounding factors in correlation analysis, Logistic stepwise regression was performed, using EGFR mutation status as dependent variable, factors including age, gender, smoking history, pathological types (set dummy variable, adenocarcinoma as reference), pathological stage and gender*smoking (interaction effect) as independent variables. Results showed that only smoking history and pathological types were correlated with EGFR mutation status. EGFR mutation occurred less frequent in patients with smoking history, odds ratio was 0.26 (95\%CI: 0.15-0.45); EGFR mutation rate was lower in squamous carcinoma patients than in adenocarcinoma patients, odds ratio was 0.15 (95\% CI: $0.05-0.47)$, as shown in Table 4.

\section{Correlation analysis between EGFR mutation types and NSCLC characteristics}

Moreover, we analyzed the correlation between EGFR mutation types and NSCLC characteristics in EGFR mutated patients. Since sample size of mutation types except for 19-del and L858R was small, they were merged into one group-“others". As shown in Table 3, only pathological type was found relative with EGFR mutation types $(P=0.02)$. No significant difference was found among the three groups: 19-del, L858R and others, with respect to age, gender, smoking history and pathological stages 
Table 3. EGFR mutation in Subgroups Classified by NSCLC Characteristics

\begin{tabular}{|c|c|c|c|c|c|c|c|c|}
\hline & \multicolumn{4}{|c|}{ All NSCLC patients } & \multicolumn{4}{|c|}{ EGFR mutated NSCLC patients } \\
\hline & Total & $\begin{array}{l}\text { EGFR } \\
\text { mutated }\end{array}$ & $\begin{array}{l}\text { EGFR wild- } \\
\text { type }\end{array}$ & $\mathrm{P}$ & 19-del & L858R & Others* & $\mathrm{p}$ \\
\hline $\begin{array}{l}\text { Age }(\text { year, } \\
\text { Mean } \pm \text { SD) }\end{array}$ & $58.31 \pm 11.45$ & $58.82 \pm 10.64$ & $57.83 \pm 12.18$ & 0.486 & $56.61 \pm 12.53$ & $60.65 \pm 8.62$ & $59.18 \pm 10.56$ & 0.144 \\
\hline \multicolumn{9}{|l|}{ Gender $(\mathrm{N}, \%)$} \\
\hline Female & $113(43.3 \%)$ & $65(57.5 \%)$ & $48(42.5 \%)$ & \multirow[t]{2}{*}{0.012} & $23(35.4 \%)$ & $30(46.2 \%)$ & $12(18.5 \%)$ & \multirow[t]{2}{*}{0.089} \\
\hline Male & $148(56.7 \%)$ & $62(41.9 \%)$ & $86(58.1 \%)$ & & $19(30.6 \%)$ & $21(33.9 \%)$ & $22(35.5 \%)$ & \\
\hline \multicolumn{9}{|c|}{ Smoking history (N, \%) } \\
\hline $\begin{array}{l}\text { Former or current } \\
\text { smoker }\end{array}$ & $109(41.8 \%)$ & $33(30.3 \%)$ & $76(69.7 \%)$ & \multirow[t]{2}{*}{$<0.001$} & $8(24.2 \%)$ & $13(39.4 \%)$ & $12(36.4 \%)$ & \multirow[t]{2}{*}{0.274} \\
\hline No smoker & $152(58.2 \%)$ & $94(61.8 \%)$ & $58(38.2 \%)$ & & $34(36.2 \%)$ & $38(40.4 \%)$ & $22(23.4 \%)$ & \\
\hline \multicolumn{9}{|c|}{ Pathological types (N, \%) } \\
\hline Adenocarcinoma & $227(87.0 \%)$ & $120(52.9 \%)$ & $107(47.1 \%)$ & \multirow{3}{*}{$<0.001$} & $41(34.2 \%)$ & $51(42.5 \%)$ & $28(23.3 \%)$ & \multirow{3}{*}{0.002} \\
\hline $\begin{array}{l}\text { Squamous } \\
\text { carcinoma }\end{array}$ & $27(10.3 \%)$ & $4(14.8 \%)$ & $23(85.2 \%)$ & & $1(25.0 \%)$ & $0(0.0 \%)$ & $3(75.0 \%)$ & \\
\hline $\begin{array}{l}\text { Adenosquamous } \\
\text { carcinoma }\end{array}$ & $7(2.7 \%)$ & $3(42.9 \%)$ & $4(57.1 \%)$ & & $0(0.0 \%)$ & $0(0.0 \%)$ & $3(100.0 \%)$ & \\
\hline \multicolumn{9}{|c|}{ Pathological stage (N, \%) } \\
\hline \multicolumn{9}{|l|}{ P stage } \\
\hline I & $25(9.6 \%)$ & $13(52.0 \%)$ & $12(48.0 \%)$ & \multirow{4}{*}{0.981} & $5(38.5 \%)$ & $5(38.5 \%)$ & $3(23.1 \%)$ & \multirow{4}{*}{0.182} \\
\hline II & $21(8.0 \%)$ & $10(47.6 \%)$ & $11(52.4 \%)$ & & $2(20.0 \%)$ & $6(60.0 \%)$ & $2(20.0 \%)$ & \\
\hline III & $74(28.4 \%)$ & $35(47.3 \%)$ & $39(52.7 \%)$ & & $6(17.1 \%)$ & $16(45.7 \%)$ & $13(37.1 \%)$ & \\
\hline IV & $141(54.0 \%)$ & $69(48.9 \%)$ & $72(51.1 \%)$ & & $29(42.0 \%)$ & $24(34.8 \%)$ & $16(23.2 \%)$ & \\
\hline \multicolumn{9}{|l|}{ T stage } \\
\hline $\mathrm{T} 1$ & $51(19.5 \%)$ & $24(47.1 \%)$ & $27(52.9 \%)$ & \multirow{4}{*}{0.23} & $8(33.3 \%)$ & $10(41.7 \%)$ & $6(25.0 \%)$ & \multirow{4}{*}{0.906} \\
\hline $\mathrm{T} 2$ & $92(35.2 \%)$ & $44(47.8 \%)$ & $48(52.2 \%)$ & & $15(34.1 \%)$ & $17(38.6 \%)$ & $12(27.3 \%)$ & \\
\hline $\mathrm{T} 3$ & $53(20.3 \%)$ & $21(39.6 \%)$ & $32(60.4 \%)$ & & $6(28.6 \%)$ & $7(33.3 \%)$ & $8(38.1 \%)$ & \\
\hline $\mathrm{T} 4$ & $65(24.9 \%)$ & $38(58.5 \%)$ & $27(41.5 \%)$ & & $13(34.2 \%)$ & $17(44.7 \%)$ & $8(21.1 \%)$ & \\
\hline \multicolumn{9}{|l|}{$\mathrm{N}$ stage } \\
\hline No & $65(24.9 \%)$ & $33(50.8 \%)$ & $32(49.2 \%)$ & \multirow{4}{*}{0.951} & $13(39.4 \%)$ & $14(42.4 \%)$ & $6(18.2 \%)$ & \multirow{4}{*}{0.908} \\
\hline N1 & $29(11.1 \%)$ & $13(44.8 \%)$ & $16(55.2 \%)$ & & $4(30.8 \%)$ & $5(38.5 \%)$ & $4(30.8 \%)$ & \\
\hline N2 & $119(45.6 \%)$ & $57(47.9 \%)$ & $62(52.1 \%)$ & & $18(31.6 \%)$ & $23(40.4 \%)$ & $16(28.1 \%)$ & \\
\hline N3 & $48(18.4 \%)$ & $24(50.0 \%)$ & $24(50.0 \%)$ & & $7(29.2 \%)$ & $9(37.5 \%)$ & $8(33.3 \%)$ & \\
\hline \multicolumn{9}{|l|}{ M stage } \\
\hline M0 & $126(48.3 \%)$ & $61(48.4 \%)$ & $65(51.6 \%)$ & \multirow[t]{2}{*}{0.939} & $15(24.6 \%)$ & $28(45.9 \%)$ & $18(29.5 \%)$ & \multirow[t]{2}{*}{0.146} \\
\hline M1 & $135(51.7 \%)$ & $66(48.9 \%)$ & $69(51.1 \%)$ & & $27(40.9 \%)$ & $23(34.8 \%)$ & $16(24.2 \%)$ & \\
\hline
\end{tabular}

PS: * Represent other mutations, including 20-ins (3 insertions in exon 20), G719X (including G719S, G719A, G719C), L861Q, S7681, T790M and double mutation

Table 4. Logistic Stepwise Regression Analysis of EGFR Mutation and NSCLC Characteristics

\begin{tabular}{lcccccc}
\hline Independent variable & B & S.E & Wals & df & Sig. & Exp (B) (95\%CI) \\
\hline Smoking history & -1.331 & 0.275 & 23.459 & 1 & 0.000 & $0.264(0.154-0.453)$ \\
Pathological types & & & 11.07 & 2 & 0.004 & \\
Squamous carcinoma & -1.877 & 0.574 & 10.701 & 1 & 0.001 & $0.153(0.050-0.471)$ \\
Adenosquamous carcinoma & -0.612 & 0.807 & 0.575 & 1 & 0.448 & $0.542(0.111-2.638)$ \\
\hline
\end{tabular}

Table 5. EGFR Mutation Types and Pathological Types of EGFR Mutated NSCLC Patients

\begin{tabular}{lcccc}
\hline EGFR mutation type & adenocarcinoma & Non-adenocarcinoma & $P$ & OR(95\%CI) \\
\hline 19-del & $41(34.2 \%)$ & $1(14.3 \%)$ & $0.452^{*}$ & $/$ \\
L858R & $51(42.5 \%)$ & $0(0.0 \%)$ & $0.003^{* *}$ & $1.214(1.040-1.418)$ \\
others & $28(23.3 \%)$ & $6(85.7 \%)$ & $0.041^{* * *}$ & $/$ \\
Total & 120 & 7 & 0.001 & \\
\hline
\end{tabular}

PS:*19-del vs L858R; ** L858R vs others; *** others vs $19-$ del; $\alpha{ }^{\prime}=\alpha / 3=0.05 / 3=0.0167$ after bonferroni correction 
(All $p>0.05$ ). Meanwhile, from the pathological stage analysis, we could also find that lymphatic metastasis (N0 vs $\mathrm{N} 1+\mathrm{N} 2+\mathrm{N} 3)\left(\mathrm{x}^{2}=1.8, P=0.40\right)$ and distant metastasis (M0 vs M1) $\left(\mathrm{x}^{2}=3.8, P=0.15\right)$ were irrelative with EGFR mutation types either.

Squamous carcinoma and adenosquamous carcinoma were merged into one group called non-adenocarcinoma due to the limited sample size. It showed that EGFR mutation type distribution was different between adenocarcinoma and non-adenocarcinoma $(p=0.00)$, 19del and L858R occurred more often in adenocarinoma patients, while other mutations occurred more in nonadenocarcinoma patients. Pairwise comparison showed that frequencies of L858R and other mutation between adenocarcinoma and non-adenocarcinoma patients were statistically different ( $p=0.00, \mathrm{OR}=1.2,95 \% \mathrm{CI}: 1.0-1.4)$, after bonferroni correction, as shown in Table 5. Of the 6 non-adenocarcinoma patients, 3 cases with T790M alone were all Adenosquamous carcinoma, while 2 cases with L858R combining T790M and 1 case with L861Q or S768I were squamous carcinoma patients.

\section{Discussion}

EGFR mutation status and mutation type are essential for EGFR-TKIs application in NSCLC patients. In this study, we investigated the EGFR mutation profile of NSCLC patients in Southwest China. Nearly half of the patients were EGFR mutated, with L858R and 19-del as dominating types. Smoking history and pathological type were independent predictors of EGFR mutation, while only pathological type was related with specific EGFR mutation type.

EGFR mutation rate varies in different countries, for instance, 22\% in USA (Sholl et al., 2015), 11.9\% in Lebanon (Naderi et al., 2015), 9.6\% in Germany (Gahr et al., 2013), 9.6\% in France (Mansuet-Lupo et al., 2014), $16.6 \%$ in Spain (Rosell et al., 2009), 53.9\% in Japan (Yoshizawa et al., 2013), 36.1\% in Korea (Koo et al., 2015), 37.7\% in South India(Matam et al., 2015), range from $10 \%$ to $20 \%$ in western world, while $30 \%-50 \%$ in Asia. EGFR mutation rate was much higher in Asian people (about 30\%) than in Caucasian people (about 10\%) (Zhou and Christiani, 2011). China is a state with vast territory and multi-nations, EGFR mutation rate varies in different regions, range from $30 \%$ to $50 \%$. For example, EGFR mutation rate was $35.3 \%$ (Zhao et al., 2014) to 45.8\% (Zheng et al., 2014) in Hunan province, 38.2\% in Ningbo city (Tian et al., 2014), 32.4\% in Shanghai, Hangzhou and Kunming cities (Dong et al., 2006), 45.8\% in Hunan province, $50.2 \%$ in 17 hospitals in mainland China (Shi et al., 2014). The mutation rate in Southwest China was $48.7 \%$ in this study. The discrepancy may be caused by reasons such as region divergence, inclusion criteria, and detection method, especially the last one. Studies presented by Zhao Jing (Zhao et al., 2014), Tian Hui (Tian et al., 2014) and Dong Gangqiang (Dong et al., 2006) were performed using Sanger sequencing method, while ARMS method was used in studies conducted by Zheng et al. (2014), Shi et al. (2014) and us, we could easily conclude that mutation rate was much higher in studies performed by ARMS than sequencing. The sensitivity of ARMS was much higher than direct sequencing, which could detect mutation as low as 0.1$1.0 \%$, and the results from ARMS were more consistent with EGFR-TKIs treatment response (Shaozhang et al., 2014). As tumor cells account for only a small part of pathological tissue, especially the pleural fluid sample, and only part of the tumor cells were EGFR mutated, choosing a hypersensitive method is essential, to detect more people who would benefit from EGFR-TKIs.

Our study showed that 19-del and L858R were the dominating mutation types, consistent with previous studies. Mutation rate of 19-del was reported higher than L858R in foreign researches (Gahr et al., 2013; Yoshizawa et al., 2013; Naderi et al., 2015), while in China, the proportion of the two mutations was controversial, some reported 19-del occurred more often, (Dong et al., 2006; Zhao et al., 2014) some showed similar occurrence rate (Tang et al., 2014; Zheng et al., 2014), and a report in Sichuan showed L858R mutation rate was higher (Dong et al., 2011). In our study, L858R mutation was found most frequently, accounted for $40.9 \%$, slightly higher than 19-del (33.1\%).

EGFR double mutation is not rare in Asia, and it may resulted in completely different bio effects due to conformation change on the previous mutant (Doss et al., 2014; Lowder et al., 2015), thus need to pay more attention. 6.9\% (7/102) double mutation rate of all mutated patients in Japan was reported (Yokoyama et al., 2006), of which patterns carrying mutations in exon 20 accounted for $57.1 \%$ (4/7); A research in China discovered 5 double mutations in 145 NSCLC patients, which were all L858R/ delE746-A750 (Zhang, 2007); 7.1\% double mutation rate of all mutated patients was found in another study (Lin et al., 2014), mutation in exon 21 was more likely to be concurrent with exon 20 mutation (resistant mutation) than 19-del (4/11 vs 1/11). In our study, double mutation rate was $10.2 \%$ (13 cases), of which L858R combined with T790M occurred most often. The mutation pattern differed from one another in above researches, maybe caused by limited sample size of double mutation, however, we could find that L858R concurrent with other mutations were more common in above studies, it might indicate that patients with exon 21 mutation was more likely to induce other mutations. It's worth mentioning that mutation pattern carrying T790M accounted for $76.9 \%$ (10/13), the combination of T790M mutation might change the structure of cytoplasmic juxtamenbrane segment (JM) or make the ligand escape from the binding pocket, leading to extending oncogenic activity and sharply sensitivity reduction (Doss et al., 2014; Lowder et al., 2015).

It was found that females, no smoker, adenocarcinoma patients had greater EGFR mutation rate, consistent with most previous studies. However, in our study, logistic stepwise regression analysis showed that only smoking history and pathological type were independent predictor of EGFR mutation, rather than gender, consistent with studies conducted by Wu YL and Shi Y (Wu et al., 2007; Shi et al., 2014), while inconsistent with Liam et al.' study, which regarded only smoking status as independent predictor (Liam et al., 2014). The difference of EGFR 
mutation rate between females and males may be caused by higher smoking rate in males. In addition, lymph nodes involvement was reported with EGFR mutation in some studies (Shi et al., 2014; Zheng et al., 2014), $P$ and $\mathrm{T}$ stages were also reported correlated with EGFR mutation (Tomita et al., 2014), while our studies indicated no association of EGFR mutation with pathological stage, lymph nodes and distant metastasis, consistent with other studies (Sahoo et al., 2011; Zhang et al., 2013; Tian et al., 2014; Zhao et al., 2014; Zheng et al., 2014), suggesting constant impact of EGFR mutation during the whole pathogenic process from the very beginning.

The mutation pattern distribution was found no significant difference with respect to age, gender, and smoking history, consistent with previous study (Gu et al., 2007), indicating that EGFR mutations in smokers carried no signatures of mutagens in cigarette smoke. Furthermore, $\mathrm{Gu}$ et al. reported no association of mutation pattern with tumor histology (Gu et al., 2007), however, in our study, 19-del and L858R were found to occur more often in adenocarcinoma patients while other mutations except for 19-del and L858R tended to occur in non-adenocarcinoma patients. Of the 6 non-adenocarcinoma patients, 3 cases with T790M alone were all adenosquamous carcinoma patients, while 2 cases with L858R combining T790M and 1 case with L861Q or S768I were squamous carcinoma patients, suggested that different mutation pattern may participate in particular pathogenesis of different NSCLC pathological types. Other mutations except for 19-del and L858R, especially the resistant mutation T790M, should be brought to attention in non-adenocarcinoma patients.

In summary, EGFR mutation rate in Southwest China was $48.7 \%$, consistent with most Asian regions, and nearly $40 \%$ patients carried sensitive mutations, who could benefit from EGFR-TKIs targeting therapy. EGFR mutation has been tested in only $9.6 \%$ patients in China, and EGFR-TKIs were used more as salvage (14.8\%) rather than upfront therapy (5.3\%) (Xue et al., 2012). Therefore, promoting EGFR mutation detection is of great value in NSCLC treatment. Furthermore, we found that only smoking history and pathological type were independent predictors for EGFR mutation. 19-del and L858R were the dominating mutation types, tending to occur more frequently in adenocarcinoma patients, while nonadenocarcinoma patients were tend to be other mutation types, especially mutation patterns containing T790M.

\section{Acknowledgements}

Funding: This study was supported by grants from the National Natural Science Foundation of China (NSFC81401727 and NSFC-81472026). The fund had no role in the study design, data collection and analysis, decision to publish, or the preparation of the manuscript.

\section{References}

Dong DD, Tang Y, Zou Y, et al (2011). Study of exons 19 and 21 mutations of epidermal growth factor receptor gene in patients with lung adenocarcinoma of Sichuan Province. Chinese J Clin Experimental Pathol, 27, 1306-9.
Dong QG, Han BH, Huang JS, et al (2006). Analysis of EGFR mutations in 176 cases of non-small cell lung cancer. Chinese J Oncol, 28, 686-90.

Doss GP, Rajith B, Chakraborty C, et al (2014). Structural signature of the G719S-T790M double mutation in the EGFR kinase domain and its response to inhibitors. Sci Rep, 4, 5868.

Eck MJ, Yun CH (2010). Structural and mechanistic underpinnings of the differential drug sensitivity of EGFR mutations in non-small cell lung cancer. Biochim Biophys Acta, 1804, 559-66.

Gahr S, Stoehr R, Geissinger E, et al (2013). EGFR mutational status in a large series of Caucasian European NSCLC patients: data from daily practice. Br J Cancer, 109, 1821-8.

Gu D, Scaringe WA, Li K, et al (2007). Database of somatic mutations in EGFR with analyses revealing indel hotspots but no smoking-associated signature. Hum Mutat, 28, 760-70.

Kobayashi S, Boggon TJ, Dayaram T, et al (2005). EGFR mutation and resistance of non-small-cell lung cancer to gefitinib. $N$ Engl J Med, 352, 786-92.

Koo DH, Kim KP, Choi CM, et al (2015). EGFR-TKI is effective regardless of treatment timing in pulmonary adenocarcinoma with EGFR mutation. Cancer Chemother Pharmacol, 75, 197-206.

Liam CK, Leow HR, How SH, et al (2014). Epidermal growth factor receptor mutations in non- small cell lung cancers in a multiethnic malaysian patient population. Asian Pac J Cancer Prev, 15, 321-6.

Lin LA, Liu ZY, Yang JS, et al (2014). Distribution and clinical significance of EGFR mutations in phase I non-small-cell lung cancer. Chinese J Thoracic Cardiovascular Surgery, 30.

Lowder MA, Doerner AE, Schepartz A (2015). Structural Differences between Wild-Type and Double Mutant EGFR Modulated by Third-Generation Kinase Inhibitors. $J$ Am Chem Soc, 137, 6456-9.

Lynch TJ, Bell DW, Sordella R, et al (2004). Activating mutations in the epidermal growth factor receptor underlying responsiveness of non-small-cell lung cancer to gefitinib. $N$ Engl J Med, 350, 2129-39.

Mansuet-Lupo A, Bobbio A, Blons H, et al (2014). The new histologic classification of lung primary adenocarcinoma subtypes is a reliable prognostic marker and identifies tumors with different mutation status: the experience of a French cohort. Chest, 146, 633-43.

Matam K, Goud I, Lakshmi MA, et al (2015). Correlation between EGFR Gene Mutations and Lung Cancer: a Hospital-Based Study. Asian Pac J Cancer Prev, 16, 7071-6.

Mitsudomi T, Kosaka T, Endoh H, et al (2005). Mutations of the epidermal growth factor receptor gene predict prolonged survival after gefitinib treatment in patients with non-smallcell lung cancer with postoperative recurrence. J Clin Oncol, 23, 2513-20.

Mok TS, Wu YL, Thongprasert S, et al (2009). Gefitinib or carboplatin-paclitaxel in pulmonary adenocarcinoma. $N$ Engl J Med, 361, 947-57.

Naderi S, Ghorra C, Haddad F, et al (2015). EGFR mutation status in Middle Eastern patients with non-squamous nonsmall cell lung carcinoma: A single institution experience. Cancer Epidemiol, 39, 1099-102.

National Office for Cancer Prevention and Control NCfCR, Disease Prevention and Control Bureau, Ministry of HealthNational Office for Cancer Prevention and Control, National Center for Cancer Registry, Disease Prevention and Control Bureau, Ministry of Health (2010). Chinese cancer mortality report: Third national retrospect spotcheck of death-causation. Beijing, China: People's Medical 
Prevalence and Clinical Profile of EGFR Mutations in Non-Small-Cell Lung Carcinoma Patients in Southwest China

Publishing House, 35.

Pao W, Hutchinson KE (2012). Chipping away at the lung cancer genome. Nat Med, 18, 349-51.

Rosell R, Moran T, Queralt C, et al (2009). Screening for epidermal growth factor receptor mutations in lung cancer. $N$ Engl J Med, 361, 958-67.

Sahoo R, Harini VV, Babu VC, et al (2011). Screening for EGFR mutations in lung cancer, a report from India. Lung Cancer, 73, 316-9.

Shaozhang Z, Ming Z, Haiyan $P$, et al (2014). Comparison of ARMS and direct sequencing for detection of EGFR mutation and prediction of EGFR-TKI efficacy between surgery and biopsy tumor tissues in NSCLC patients. Med Oncol, 31, 926.

She J, Yang $P$, Hong Q, et al (2013). Lung cancer in China: challenges and interventions. Chest, 143, 1117-26.

Shepherd FA, Rodrigues Pereira J, Ciuleanu T, et al (2005). Erlotinib in previously treated non-small-cell lung cancer. N Engl J Med, 353, 123-32.

Shi Y, Au JS, Thongprasert S, et al (2014). A prospective, molecular epidemiology study of EGFR mutations in Asian patients with advanced non-small-cell lung cancer of adenocarcinoma histology (PIONEER). J Thorac Oncol, 9, 154-62.

Sholl LM, Aisner DL, Varella-Garcia M, et al (2015). Multiinstitutional Oncogenic Driver Mutation Analysis in Lung Adenocarcinoma: The Lung Cancer Mutation Consortium Experience. J Thorac Oncol, 10, 768-77.

Tang Y, Zhang L, Tan X, et al (2014). Analysis of EGFR mutations in non-small cell lung cancer in Nanning Guangxi China. J Modern Oncol.

Tian H, Kaizhong YU, Mao Z, et al (2014). Study on mutation of EGFR gene in 314 cases of non-small cell lung cancers. J Shanxi Medical University.

Tomita M, Ayabe T, Chosa E, et al (2014). Epidermal growth factor receptor mutations in Japanese men with lung adenocarcinomas. Asian Pac J Cancer Prev, 15, 10627-30.

Torre LA, Bray F, Siegel RL, et al (2015). Global cancer statistics, 2012. CA Cancer J Clin, 65, 87-108.

Wu YL, Zhong WZ, Li LY, et al (2007). Epidermal growth factor receptor mutations and their correlation with gefitinib therapy in patients with non-small cell lung cancer: a metaanalysis based on updated individual patient data from six medical centers in mainland China. J Thorac Oncol, 2, 430-9.

Xue C, Hu Z, Jiang W, et al (2012). National survey of the medical treatment status for non-small cell lung cancer (NSCLC) in China. Lung Cancer, 77, 371-5.

Yasuda H, Kobayashi S, Costa DB (2012). EGFR exon 20 insertion mutations in non-small-cell lung cancer: preclinical data and clinical implications. Lancet Oncol, 13, 23-31.

Yokoyama T, Kondo M, Goto Y, et al (2006). EGFR point mutation in non-small cell lung cancer is occasionally accompanied by a second mutation or amplification. Cancer Sci, 97, 753-9.

Yoshizawa A, Sumiyoshi S, Sonobe M, et al (2013). Validation of the IASLC/ATS/ERS lung adenocarcinoma classification for prognosis and association with EGFR and KRAS gene mutations: analysis of 440 Japanese patients. J Thorac Oncol, 8, 52-61.

Zhang G (2007). Molecular epidemiologic investigation of EGFR double mutations in Chinese non-small-cell lung cancer population and related studies on biological properyies. Doctoral Dissertation, 2007, 3.

Zhang Y, Wang Q, Han ZG, et al (2013). Differences in epidermal growth factor receptor gene mutations and relationship with clinicopathological features in NSCLC between Uygur and Han ethnic groups. Asian Pac J Cancer Prev, 14, 2879-83.
Zhao J, Tian Q, Huang Y, et al (2014). An Analysis of the EGFR gene mutation in 238 patients with non-small cell lung cancer in hunan province. Anti-tumor Pharmacy.

Zheng J, Xie G, Jiao LI, et al (2014). Clinical significance of EGFR mutations in non-small cell lung cancer. Chinese $J$ Clin Oncol, 41, 904-7.

Zhou W, Christiani DC (2011). East meets West: ethnic differences in epidemiology and clinical behaviors of lung cancer between East Asians and Caucasians. Chin J Cancer, 30, 287-92. 